\title{
Modeling the Influence of a Magnetomechanical Effect on the Permeability Tensor of a Tensductor Core
}

\author{
Roman Szewczyk $^{1, *}{ }^{\circledR}$, Michał Nowicki $^{1}{ }^{\circledR}$, Anna Ostaszewska-Liżewska $^{1}\left(\mathbb{D}\right.$ and Mika Malinen ${ }^{2}$ \\ 1 Warsaw University of Technology, Institute of Metrology and Biomedical Engineering, Boboli 8, \\ 02-525 Warsaw, Poland; m.nowicki@mchtr.pw.edu.pl (M.N.); a.ostaszewska@mchtr.pw.edu.pl (A.O.-L.) \\ 2 CSC-IT Center for Science, P.O. Box 405, FI-02101 Espoo, Finland; Mika.Malinen@csc.fi \\ * Correspondence: szewczyk@mchtr.pw.edu.pl; Tel.: +48-609-464-741
}

Received: 16 November 2019; Accepted: 1 December 2019; Published: 4 December 2019

\begin{abstract}
This paper presents a new method of modeling the influence of mechanical stresses on a magnetic permeability tensor of soft magnetic materials. The proposed method utilizes the principal stresses concept to compensate the influence of shear stresses. As a result, the stress dependence of a magnetic permeability tensor may be assessed with only the knowledge about the influence of axial stresses on magnetic properties of isotropic material. The proposed method was used for a finite element method based model of a tensductor designed for measurements of tensile forces. Due to the fact that 2D stresses distribution occurs in a tensductor, simplification of both principal stresses and a magnetic permeability tensor rotation procedure was proposed. As a result, good agreement was reached between the results of modeling and the results of experimental tests. This result validates the possibility of utilization of the proposed modeling method for the design of magnetomechanical devices.
\end{abstract}

Keywords: magnetoelastic effect; soft magnetic materials; amorphous alloys; principal stresses

\section{Introduction}

Magnetoelastic phenomenon [1,2] is connected with the significant changes of magnetic permeability of magnetic materials subjected to the influence of mechanical stresses [3]. Known also as the Villari or inverse magnetostriction effect, it is one of the better known manifestations of wide range of magnetostriction-related phenomena [4,5]. This effect has great technical importance, such as the possibility of utilization of magnetoelastic effect in the development of robust mechanical stress and force sensors for industrial automation [6-8], biomedical engineering [9], and security [10]. On the other hand, an uncontrolled magnetoelastic effect may lead to degradation of magnetic properties of inductive cores made of magnetic materials accidentally subjected to mechanical stresses. In such a case, a magnetoelastic phenomenon may lead to a decrease of efficiency of switching mode power supplies [11] or a reduction of accuracy of the operation of fluxgate sensors [12].

In spite of the importance of the magnetoelastic effect for the development of devices with cores made of soft magnetic materials, knowledge about its physical background and mathematical model description is still intensively investigated [13,14]. Existing models are mostly focused on the influence of mechanical stresses parallel [15] or perpendicular [16] to the direction of magnetization of magnetic material. The complete description of the stress dependence of a magnetic permeability tensor seems to be still not presented in the literature.

The modeling concept presented in the paper is filling this gap. This method of modeling the stress dependence of a permeability tensor in two-dimensional systems may be utilized in a finite element method (FEM) modeling of magnetic devices with flat magnetic cores, or with cores which exhibit planar symmetry. An example of such a device is a tensductor [17], the tensile stress sensor 
utilizing changes of flux density of a ribbon made of amorphous alloy for measurements of tensile forces. Moreover, the same concept may be used for modeling the characteristics of pressductors [18] introduced by the Assea company, and now widely used for large compressive force measurements in industrial automation. In the case of these sensors, the measurement characteristic is not determined by the changes of dimensions of sensors under mechanical stresses, but by the changes of the material's magnetic permeability tensor influenced by stress-induced magnetoelastic anisotropy. It should be highlighted that, until now, it was not possible to perform modeling of the output characteristics of such sensors with the finite element method, which significantly limited possibilities to optimize the sensor's performance during the development process.

\section{Modeling the Magnetoelastic Effect in 2D Systems}

Magnetoelastic characteristics of soft magnetic materials for the mechanical stresses applied in the same direction as magnetizing fields were widely presented in the literature [19]. Under the influence of axial tensile stresses $\sigma$ in the elastic region, the permeability of soft magnetic material with a positive saturation magnetostriction constant $\lambda_{\mathrm{s}}$ increases, whereas for material with negative saturation magnetostriction the permeability decreases [20].

In the case of mechanical stresses $\sigma_{\perp}$ perpendicular to magnetization direction [21], it was confirmed [22] that effective stresses $\sigma_{e}$ influencing the magnetic properties may be assessed from the equation:

$$
\sigma_{e}=-v \cdot \sigma_{\perp}
$$

where $\sigma_{\perp}$ is the value of stress perpendicular to the magnetizing axis direction and $v$ is Poisson's ratio. Considering this assumption, the $\mathrm{X}-\mathrm{Y}$ permeability tensor $\mu$ of isotropic material with the positive saturation magnetostriction $\lambda_{S}$ subjected to tensile stresses $\sigma$ in $\mathrm{X}$ direction is presented in Figure 1 using polar coordinate parameterization. It should be highlighted that, for such permeability tensor $\mu$, the flux density vector $B$ in the material is not always perpendicular to the magnetizing field $H$, which is crucial from the point of view of principles of operation of tensductors and pressductors.

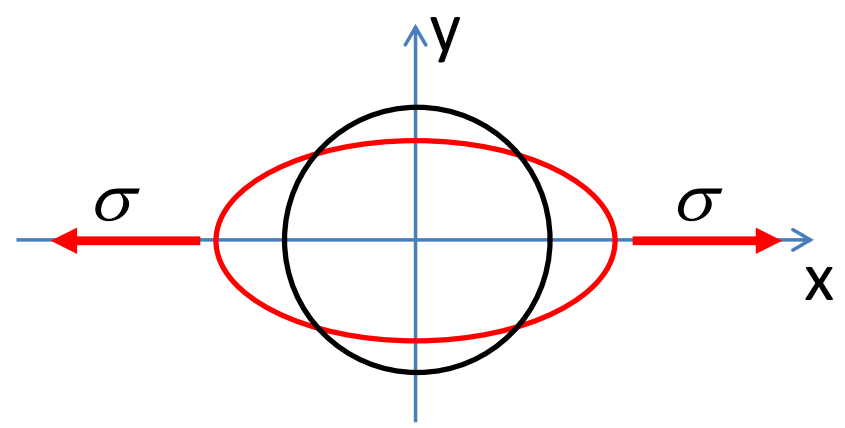

Figure 1. Permeability tensor $\mu$ in polar coordinate system: isotropic material (black line), magnetic materials with the positive saturation magnetostriction $\lambda_{s}$ subjected to tensile stresses $\sigma$ in $\mathrm{X}$ direction (red line).

As it was proven experimentally, soft magnetic materials are strongly sensitive not only to tensile or compressive stresses, but also to shear stresses [23]. However, it is very sophisticated to assess simultaneous interaction of axial stresses $\sigma$ and shear stresses $\tau$ on properties of soft magnetic material. To overcome this problem, principal stresses concept was used [24].

As it is presented in Figure 2a,b, the tensile or compressive stress $\tau$ and shear stress $\tau$ system can be substituted by the axial stresses with respect to axes that make an angle $\varphi$ with the original axes. These stresses are called principal stresses and enable avoidance of shear stresses $\tau$ in magnetoelastic analyses. 


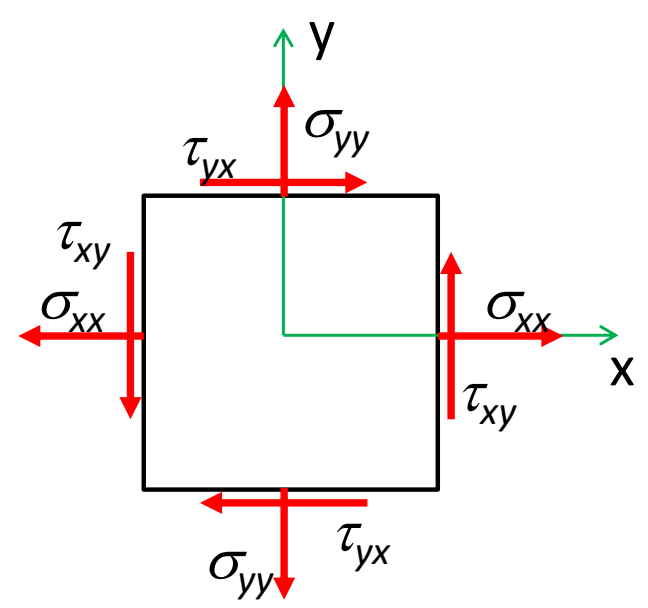

(a)

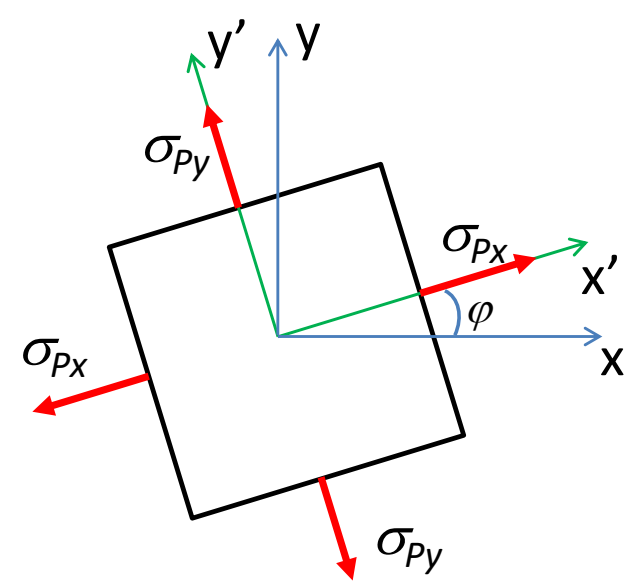

(b)

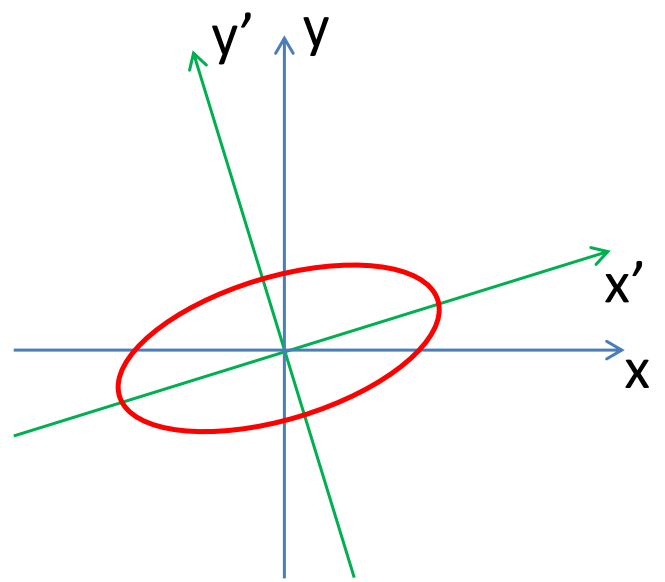

(c)

Figure 2. Stress dependence of a permeability tensor $\mu$ in 2D system: (a) a combination of axial and shear stresses $\sigma$ and $\tau,(\mathbf{b})$ principal stresses $\sigma_{P}$ representing the same state of stress, (c) representation of permeability tensor $\mu$ depending on the principal stresses $\sigma_{P}$.

It should be highlighted that also the stress dependence of the permeability tensor may be analyzed considering principal stresses. As a result, the permeability tensor (with uniaxial anisotropy) should 
be rotated in X-Y plane by the angle $\varphi$ determined by the principal stresses. Such a rotated magnetic permeability tensor in a planar coordinate system is presented in Figure 2c.

The magnetic relative permeability tensor $\mu_{u}$ with uniaxial anisotropy with main axes oriented in the direction of $\mathrm{X}, \mathrm{Y}$, and $\mathrm{Z}$ axes is given as

$$
\mu_{u}=\left[\begin{array}{ccc}
\mu_{x x} & 0 & 0 \\
0 & \mu_{y y} & 0 \\
0 & 0 & \mu_{z z}
\end{array}\right]
$$

The stress dependence (in elastic region) of magnetic permeability $\mu$ of isotropic magnetic material for stresses applied in the same direction as the magnetizing field $H$ is given by the $\mu(\sigma)$ dependence presented previously in the literature [2,3]. However, for the 2D stress distribution, considering the Equation (1), the effective stresses $\sigma_{x x}$ and $\sigma_{y y}$ in $\mathrm{X}$ and $\mathrm{Y}$ direction can be calculated from the following equations:

$$
\begin{aligned}
& \sigma_{x x}=\sigma_{x}-v \cdot \sigma_{y}, \\
& \sigma_{y y}=\sigma_{y}-v \cdot \sigma_{x} .
\end{aligned}
$$

Finally, the parameters of the magnetic permeability tensor in $X$ and $Y$ directions can be calculated as

$$
\begin{aligned}
& \mu_{x x}=\mu\left(\sigma_{x x}\right), \\
& \mu_{y y}=\mu\left(\sigma_{y y}\right),
\end{aligned}
$$

whereas changes of $\mu_{\mathrm{zz}}$ may be neglected, due to the fact that the sample is magnetized in $X$ and $Y$ directions as a $2 \mathrm{D}$ system.

To compensate shear stresses $\tau$, the coordinate system for representing the magnetic permeability tensor should first be aligned with the directions of the principal stresses via the rotation by the angle $\varphi$, so as to replace the axial stresses $\sigma_{x}$ and $\sigma_{y}$ by the principal stress components $\sigma_{P x}$ and $\sigma_{P y}$ in the rotated coordinate system. The components of the magnetic permeability tensor can be transformed to the components with respect to the original frame in terms of the rotation matrix $R$ [25]:

$$
R=\left[\begin{array}{ccc}
\cos (\varphi) & -\sin (\varphi) & 0 \\
\sin (\varphi) & \cos (\varphi) & 0 \\
0 & 0 & 1
\end{array}\right]
$$

The magnetic permeability tensor $\mu_{u}$ subjected to the influence of principal stresses $\sigma_{P x}$ and $\sigma_{P y}$ can thus be calculated as

$$
\mu_{u}=R \times\left[\begin{array}{ccc}
\mu\left(\sigma_{P x x}\right) & 0 & 0 \\
0 & \mu\left(\sigma_{P y y}\right) & 0 \\
0 & 0 & \mu_{z z}
\end{array}\right] \times R^{-1},
$$

where $\sigma_{P x x}$ and $\sigma_{P y y}$ are calculated according to the Equations (3)-(6).

\section{Implementation of the Model}

A tensductor sensor consists of a thin layer of amorphous alloy ribbon core and two perpendicularly oriented coils: a driving coil and a sensing coil [17] as it is presented in Figure 3. The magnetizing coil is connected to a sinewave current source with a frequency of $1 \mathrm{kHz}$ to generate the magnetizing field. Due to the fact that the thickness of the core of the tensductor is $30 \mu \mathrm{m}$, the influence of eddy currents may be neglected. For isotropic magnetic material, the flux density $B$ component in the direction of the sensing coil is equal to zero. However, when stress-induced axial anisotropy (in $\mathrm{x}$ axis direction) appears in the core of the sensor as a result of an external tensile force $F$ applied uniformly by special 
clamps [17], a component of the flux density $B$ in the direction of the sensing coil axis appears. As a result, the value of the tensile force $F$ can be measured by the measurements of voltage induced on the sensing coil.

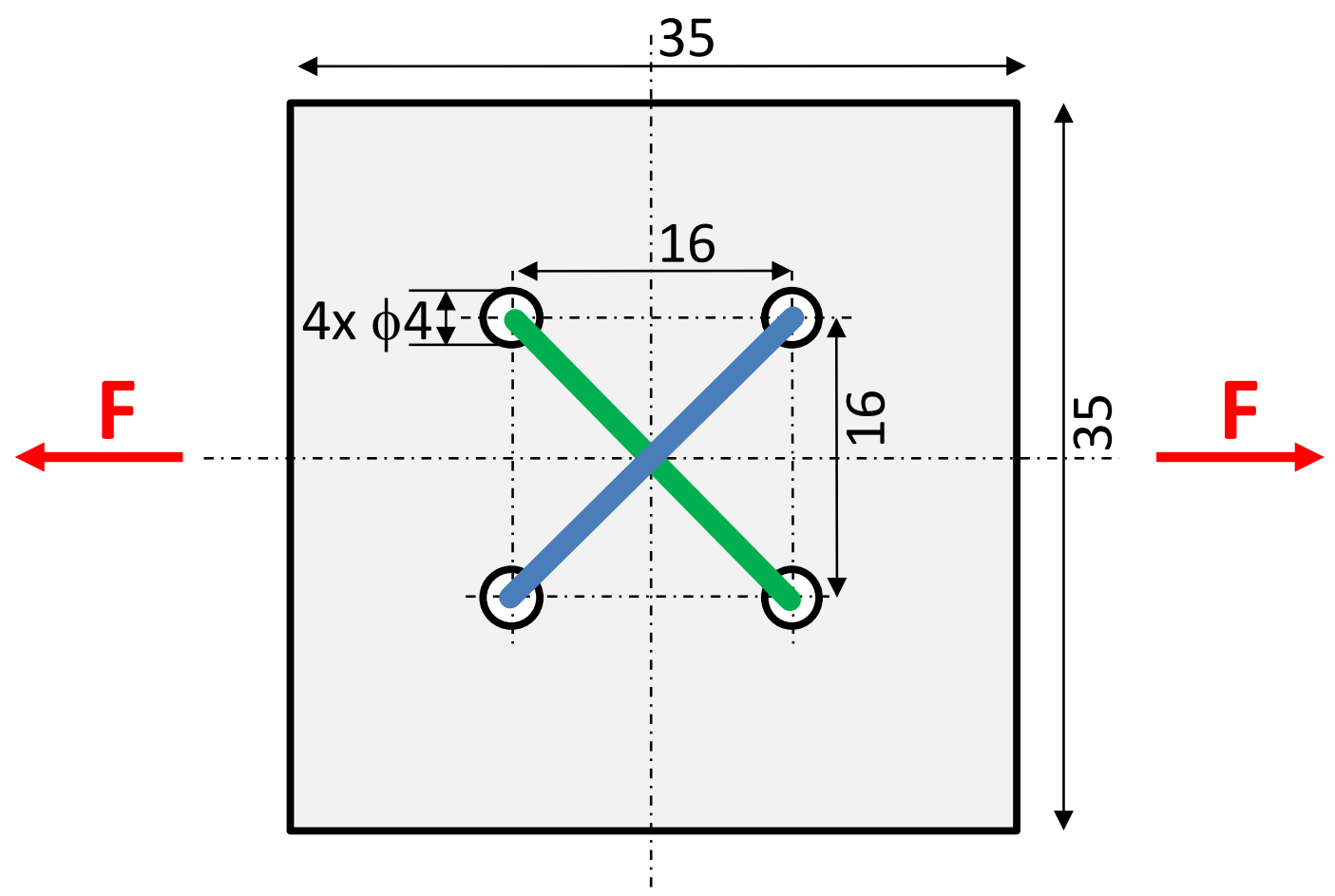

Figure 3. Schematic presentation of the proposed tensductor sensor with a core made of a square-shaped amorphous alloy ribbon; the magnetizing winding (blue), the sensing winding (green).

The proposed model of the generalization of 2D stress dependence of the magnetic permeability tensor was implemented in MATC language in Elmer FEM open-source environment [26] for finite element modeling. Meshes of tetrahedral elements were created using the NETGEN open-source mesh generator. The Delaunay method of meshing was applied [27]. With the use of Elmer FEM it was possible to estimate mechanical stress distribution in the sensor core and connect it with the magnetic permeability tensor distribution.

For the presented modeling linear relative permeability dependence $\mu_{r}(\sigma)$ for axial stresses was proposed. This dependence is presented in Figure 4 and is given by the equation

$$
\mu_{r}=5715+267 \cdot \sigma[\mathrm{MPa}]
$$




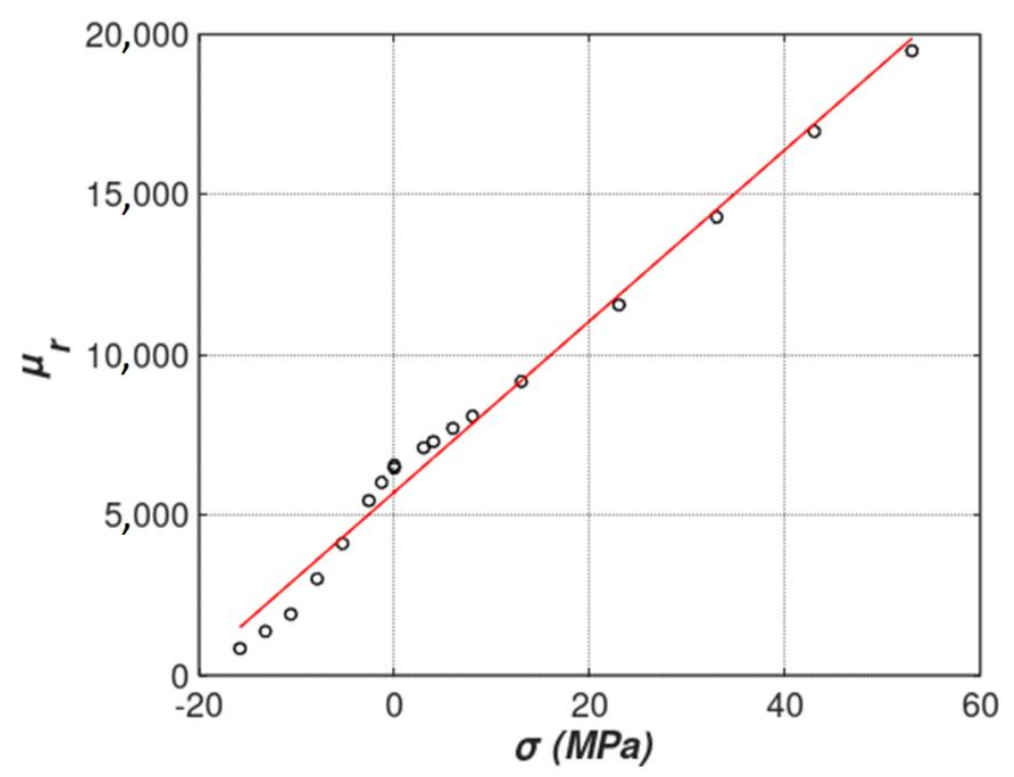

Figure 4. The dependence of relative magnetic permeability $\mu_{r}$ on the axial stress $\sigma$ in a ribbon made of $\mathrm{Fe}_{73.5} \mathrm{Cu}_{1} \mathrm{Nb}_{3} \mathrm{Si}_{15.5} \mathrm{~B}_{7}$ amorphous alloy (VITROPERM ${ }^{\circledR} 800$ ). Dependence is approximated by linear function (red). Black circles-experimental results.

The proposed dependence was estimated on the base of an experimental test for relatively small values of mechanical stresses in an elastic region of magnetoelastic characteristic. The $\mathrm{Fe}_{73.5} \mathrm{Cu}_{1} \mathrm{Nb}_{3} \mathrm{Si}_{15.5} \mathrm{~B}_{7}$ amorphous alloy was used, produced by VACUUMSCHMELZE GmbH \& Co. KG (Hanau, Germany). This material was subjected to pre-annealing in the production process and can be treated as nearly-isotropic. Measurements of hysteresis loops of samples magnetized along and across the ribbon length confirm this. The $\mathrm{Fe}_{73.5} \mathrm{Cu}_{1} \mathrm{Nb}_{3} \mathrm{Si}_{15.5} \mathrm{~B}_{7}$ amorphous alloy is a precursor of commercially available nanocrystalline VITROPERM ${ }^{\circledR} 800$ (VACUUMSCHMELZE, Hanau, Germany) [24] and was cast as ribbon $50 \mathrm{~mm}$ wide and $30 \mu \mathrm{m}$ thick. As a result, cross section of the tensductor core near its edge is equal to $1.05 \mathrm{~mm}^{2}$. The Young's modulus for these materials is around $100 \mathrm{GPa}$, with tensile strength of $1 \mathrm{GPa}$ [28]. In order to measure the magnetoelastic characteristics of this material in the elastic region, two measurement methods were used. In the first of them, the influence of tensile stress along the magnetic induction direction was measured, while in the second the tensile force and induction vectors were perpendicular (based on the method presented in [3]).

In the first test stand (Figure 5), the amorphous ribbon sample was placed inside a magnetizing Helmholtz coil, which was connected to the voltage/current converter RDM-2a (WUT, Warsaw, Poland), controlled by the arbitrary function generator (SDG1025, Siglent, Helmond, The Netherlands). The AC current induced the magnetizing field $H$, which in turn produced the magnetic induction $B$ in the sample that was measured by the sensing coil placed around the central part of the ribbon. The sensing coil was connected counter-serial with an identical air-flux compensation coil, and to the fluxmeter (model 480, Lakeshore, Westerville, OH, USA). By varying the tensile stress in the sample with suspended weights, the magnetoelastic characteristics of $B\left(\sigma_{\|}\right)$and $\mu\left(\sigma_{\|}\right)$were obtained. 


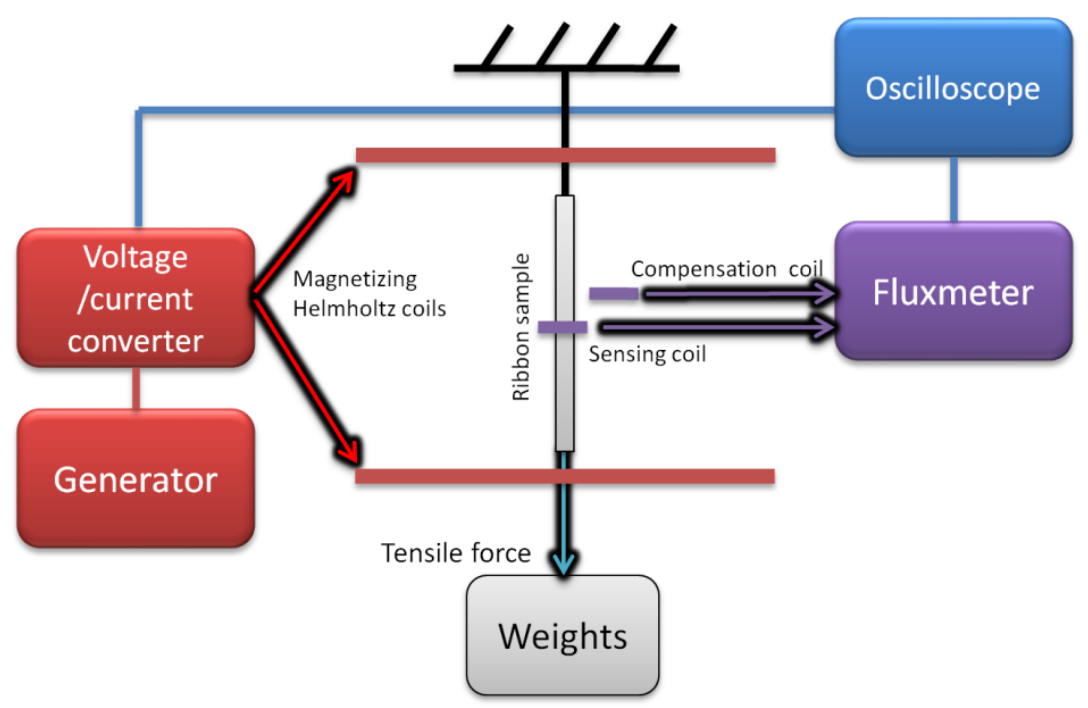

Figure 5. Schematic diagram of the measurement test stand used for the determination of magnetoelastic $B\left(\sigma_{\|}\right)$and $\mu\left(\sigma_{\|}\right)$characteristics of soft magnetic amorphous ribbon in the tensile stress region.

The second test stand (Figure 6) employed the same equipment, but the sample was a ring-shaped ribbon-wound core, wound with sensing winding, with one magnetizing rod passing through its center. The application of tensile force along the axis of the ring core produces magnetoelastic characteristics of $B\left(\sigma_{\perp}\right)$ and $\mu\left(\sigma_{\perp}\right)$, which, according to [22], after including Poisson's ratio can substitute $B \| \sigma$ magnetoelastic characteristics in the compressive stress region.

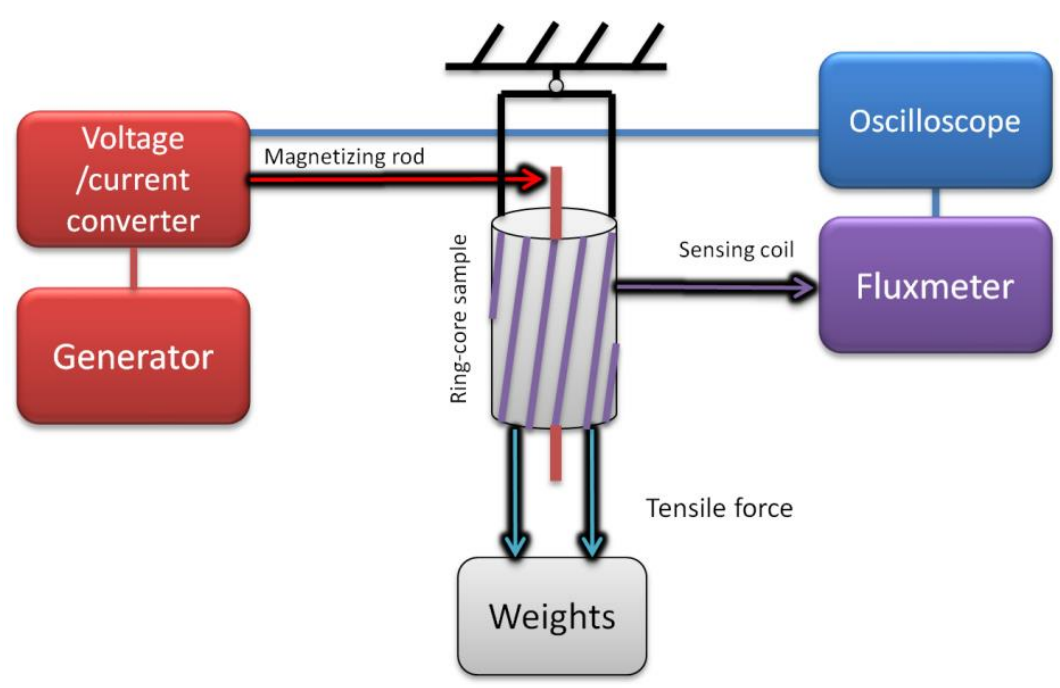

Figure 6. Schematic diagram of the measurement test stand used for the determination of magnetoelastic $B\left(\sigma_{\perp}\right)$ and $\mu\left(\sigma_{\perp}\right)$ characteristics soft magnetic amorphous ribbon in the tensile stress region.

In addition, the modeling was carried out by assuming the following mechanical parameters for the tensductor core made of $\mathrm{Fe}_{73.5} \mathrm{Cu}_{1} \mathrm{Nb}_{3} \mathrm{Si}_{15.5} \mathrm{~B}_{7}$ amorphous alloy:Young's modulus: $100 \mathrm{GPa}$; Poisson's ratio: 0.3 .

It should be also highlighted that due to high Young's modulus and mechanical hardness in the iron-based amorphous alloys, the elastic region of the stress-strain curve is observed up to the material rupture [29]. 


\section{Results of Modeling}

Figure 7 presents the results of modeling the mechanical stress distribution in the 2D system of the tensductor core, showing the axial stresses in $X$ and $Y$ direction as well as the shear stress. Figure 8 presents the same mechanical stress system in terms of the principal stresses in the coordinate system $X^{\prime}-Y^{\prime}$ obtained via rotating by the angle $\varphi$ around the $Z$ axis. It can be observed that the axial stress component corresponding to the first axis dominates in both cases. However, the stress distribution around the holes is sophisticated and may significantly influence the component of flux density $B$ responsible for the generation of voltage in the sensing coil.

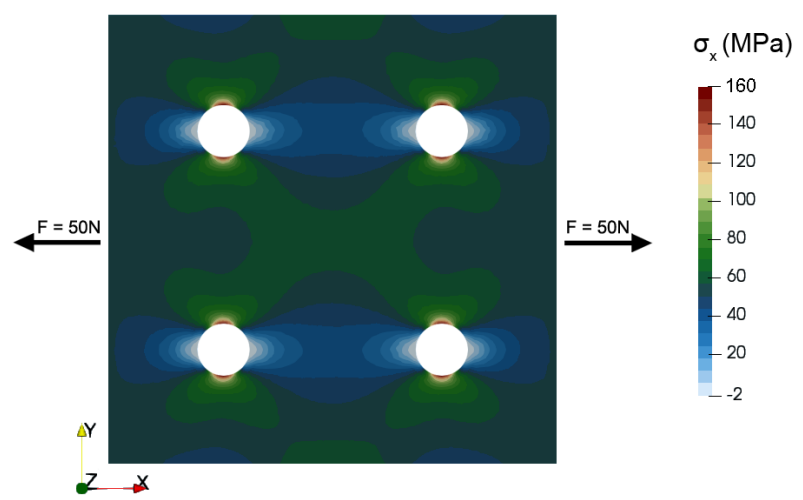

(a)

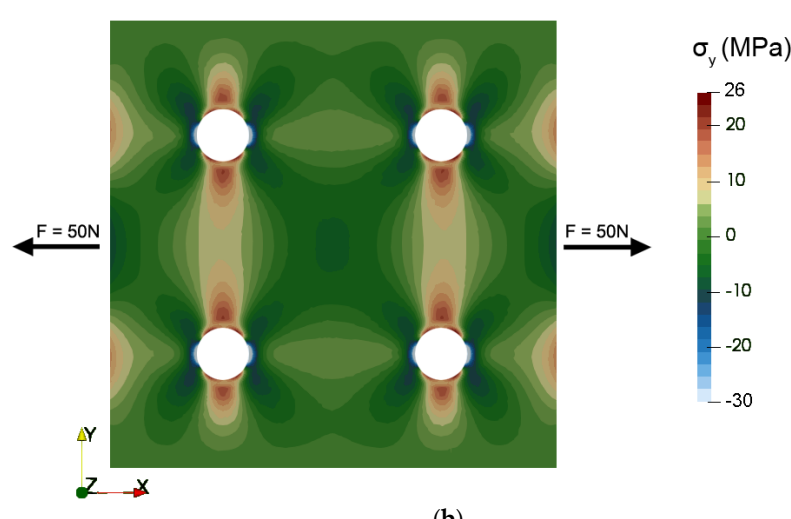

(b)

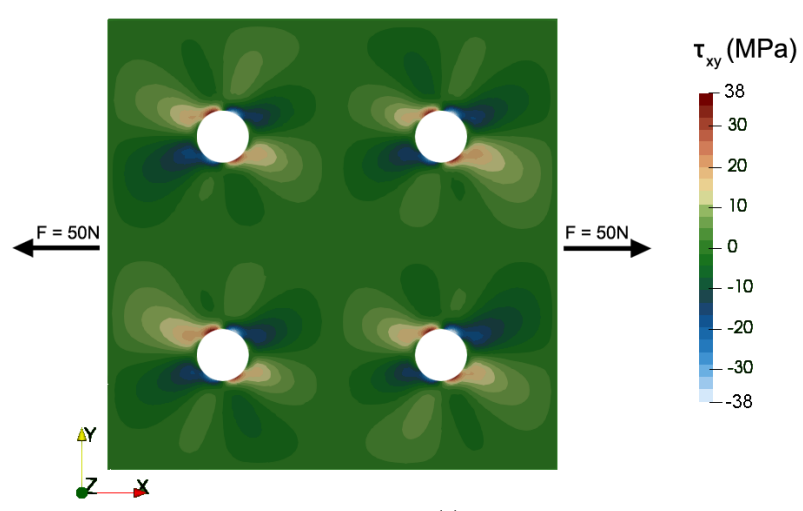

(c)

Figure 7. The results of modeling the mechanical stress distribution in the 2D system of tensductor core subjected to tensile force $F=50 \mathrm{~N}$ in $\mathrm{X}$ direction: (a) $\sigma_{x},(\mathbf{b}) \sigma_{y},(\mathbf{c}) \sigma_{x y}$. 


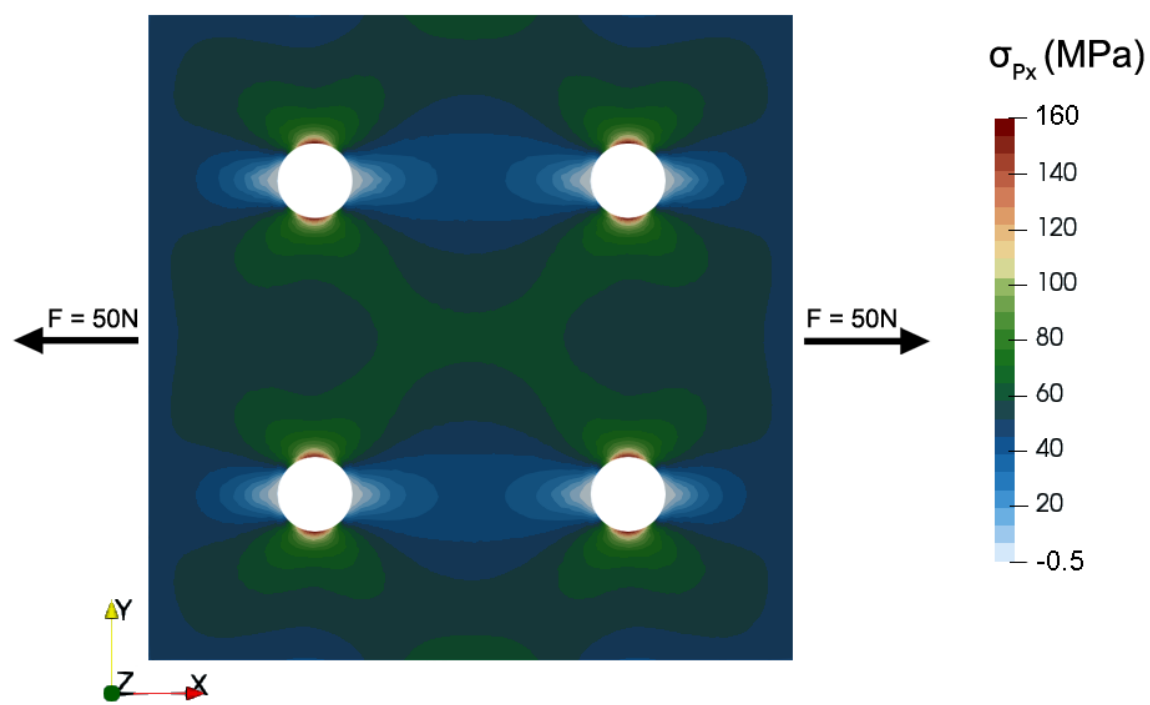

(a)
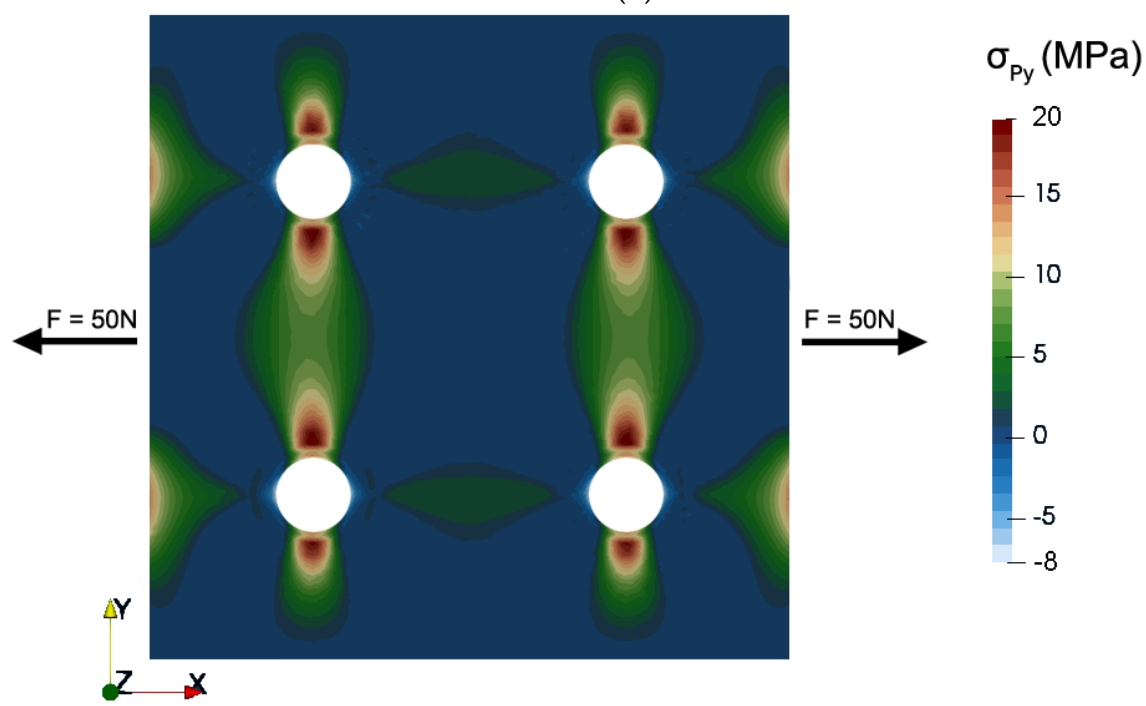

(b)

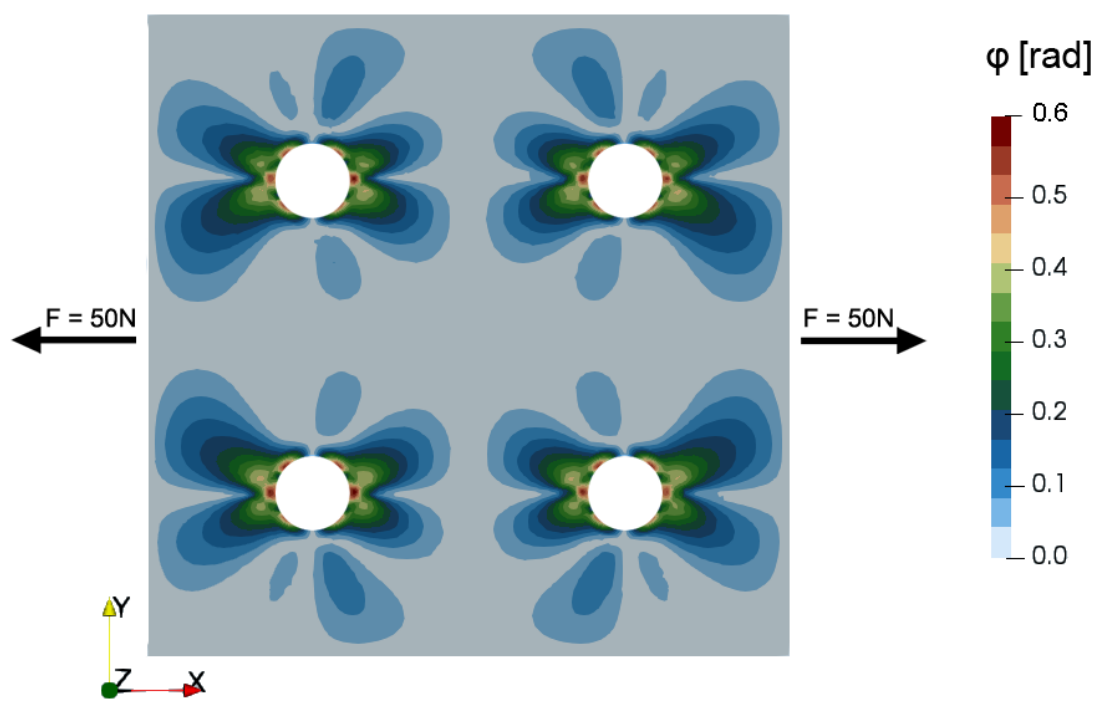

(c)

Figure 8. The results of modeling the mechanical stress distribution in terms of principal stresses for the same $2 \mathrm{D}$ system of the tensductor core subjected to tensile force $F=50 \mathrm{~N}$ in X direction: (a) $\sigma_{P x}$, (b) $\sigma_{P y},(\mathbf{c}) \varphi$. 
Figure 9 presents the flux density $B$ vector distribution in the core of the tensductor sensor. It can be seen that the mechanical stresses significantly influence the flux density $B$ vector direction by the changes of the magnetic permeability tensor. Moreover, analysis of Figure 9 enables the understanding of principles of operation of the tensductor or pressductor sensor, which is not obvious via consideration of models previously presented in the literature.

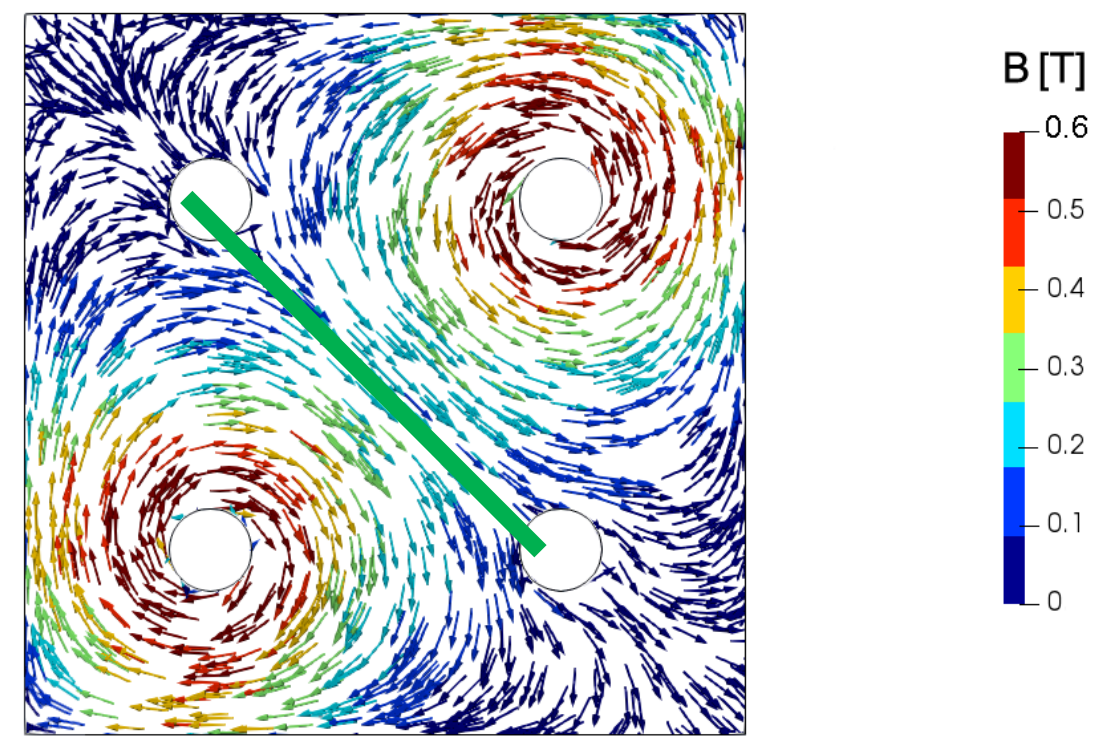

(a)

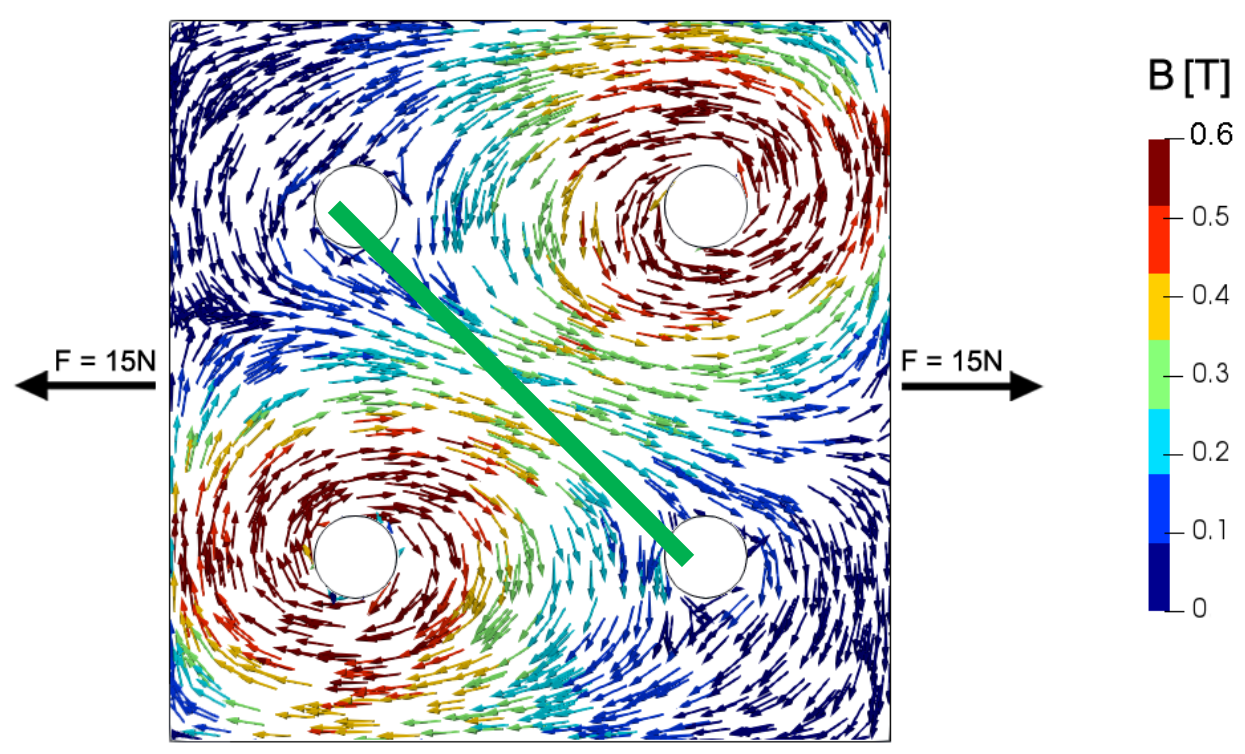

(b)

Figure 9. Flux density $B$ vector distribution in the core of tensductor sensor: (a) without stresses, (b) subjected to tensile force $F=15 \mathrm{~N}$ in $\mathrm{X}$ direction. Green line-sensing coil.

Figure 10 presents the results of modeling the characteristics of the tensductor sensor considering the magnetic flux $\phi$ responsible for the generation of the voltage in the sensing coil (the magnetic flux perpendicular to the sensing coil). It should be highlighted that the presented results of modeling are in very good agreement with experimental results presented previously in the literature [17]. As a result, the concept of modeling the stress dependence of the permeability tensor in soft magnetic materials was justified. This will enable the quantitative analyses and optimization of performance of tensductors for 
industrial applications, as well as understanding and development of other magnetoelastic sensors utilizing the cores made of soft magnetic materials.

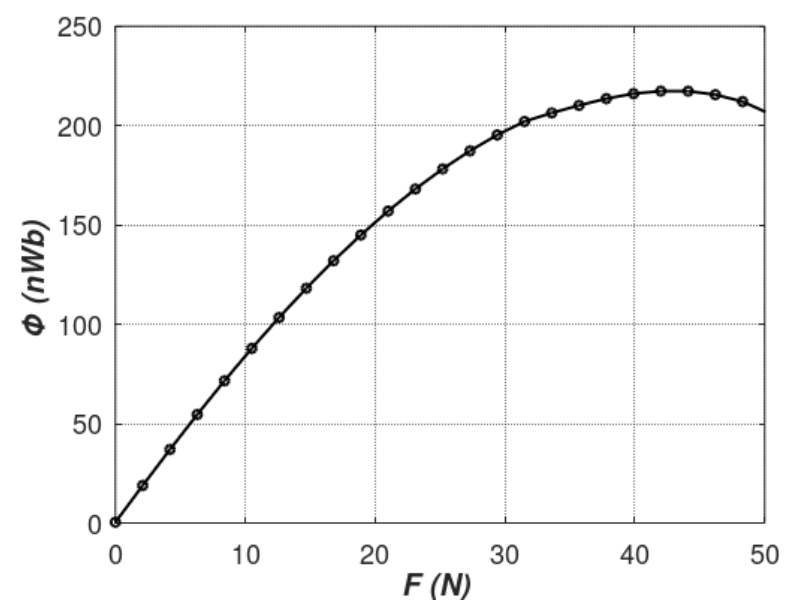

Figure 10. The results of modeling the characteristics of tensductor sensor considering the tensile force $F$ dependence of magnetic flux $\phi$ responsible for the generation of voltage in the sensing coil.

\section{Experimental Verification of the Results}

In order to compare modeling results with actual tensductor characteristics, the sensor of the same geometry and material was produced and tested on the measurement test stand (Figure 11). Instead of the first harmonic of induced voltage originally proposed for the output of this type of sensor [17], total AC magnetic flux in the sensing coil was measured by means of a sensitive fluxmeter (480, Lakeshore, Westerville, $\mathrm{OH}$, USA).

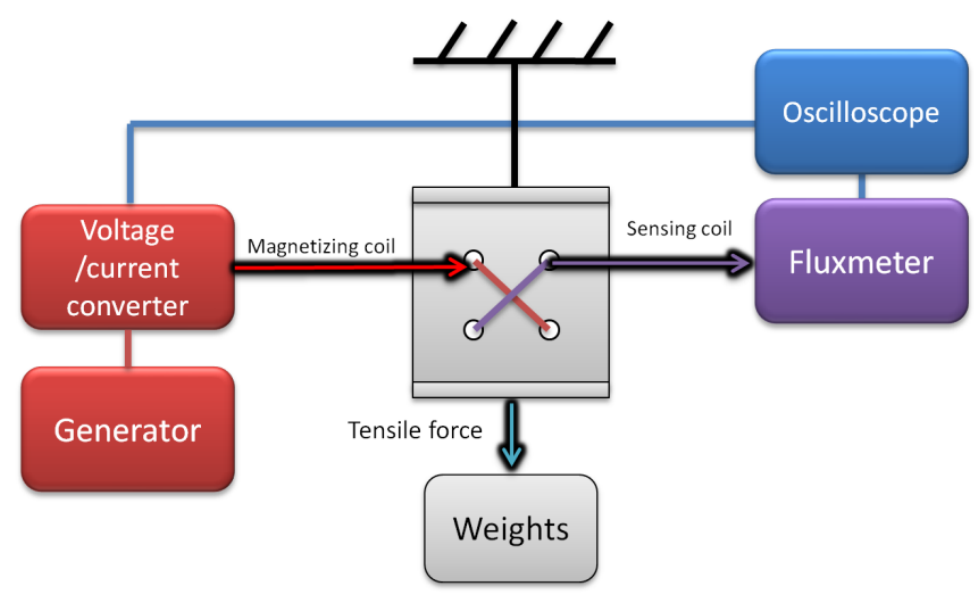

Figure 11. Schematic diagram of tensductor characterization test stand.

The results of the magnetic flux measured by the sensing coil for incrementally raised tensile force are presented in Figure 12. 


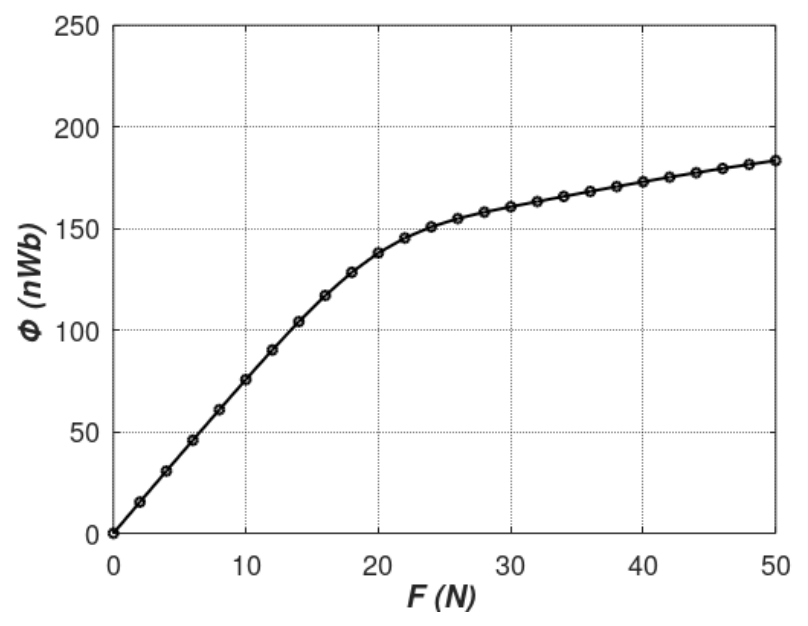

Figure 12. Graph of $B(F) / B(\sigma)$ characteristics of investigated tensductor tensile force sensor.

As can be seen, good correlation with modeling data was achieved for the range $0-20 \mathrm{~N}$ of tensile forces. However, for stresses generated by forces higher than $20 \mathrm{~N}$, the linear dependence presented by the Equation (7) seems to be not valid. This phenomenon is connected with the fact that, for higher values of mechanical stresses, the value of saturation magnetostriction of magnetic materials changes significantly [30]. As a result, Villari reversal can be observed [1,2] overruling the linear $\mu(\sigma)$ dependence. In addition, forces higher than $50 \mathrm{~N}$ could not be applied due to the sensor's mechanical integrity limitations described in [17].

\section{Conclusions}

The paper presents a new concept of modeling the stress dependence of a magnetic permeability tensor and opens new possibilities of modeling the magnetoelastic devices with the finite element method. It should be highlighted that the proposed solution technique may be successfully implemented in both commercial and open-source modeling environments, enabling the tensor description of magnetic properties of soft magnetic materials.

The proposed concept to describe the stress dependence of a magnetic permeability tensor was successfully validated experimentally on the base of measured results of the stress dependence of magnetic properties of $\mathrm{Fe}_{73.5} \mathrm{Cu}_{1} \mathrm{Nb}_{3} \mathrm{Si}_{15.5} \mathrm{~B}_{7}$ soft amorphous alloy, which were used for the modeling of tensductor characteristics. These results confirm the correctness of the proposed model for stresses created by tensile forces up to $20 \mathrm{~N}$, which is sufficient from an engineering point of view.

As a result, the proposed concept of modeling the stress dependence of a magnetic permeability tensor can be utilized in the stress-dependent models of inductive components of mechatronics devices. It should be highlighted that the source code for modeling is available under open-source license at www.github.com/romanszewczyk/FEM in the subdirectory "tensductor" for further development and validation.

The main limitation of the method is a lack of magnetic hysteresis reproduction capabilities, which leads to some level of inaccuracy of the method. Future researches will be focused on the implementation of the Preisach [13] or Jiles-Atherton [14] vector-based model of magnetic hysteresis considering the proposed methodology.

Supplementary Materials: The source code used for modeling is available at www.github.com/romanszewczyk/ FEM in the subdirectory "tensductor". Source code and binaries of ELMER FEM and NETGEN are available at web pages: https://www.csc.fi/web/elmer and https://ngsolve.org/, respectively.

Author Contributions: Conceptualization, R.S., M.N., and A.O.-L.; methodology, R.S., M.N., and A.O.-L.; software, R.S., A.O.-L., and M.M.; validation, R.S. and M.N.; formal analysis, R.S., M.N., A.O.-L., and M.M.; data curation, R.S., M.N., and A.O.-L.; writing—original draft preparation, R.S., M.N., A.O.-L., and M.M.; writing-review and editing, R.S., M.N., A.O.-L., and M.M.; visualization, A.O.-L.; supervision, R.S. 
Funding: This research was fully supported by the statutory funds of the Institute of Metrology and Biomedical Engineering, WUT.

Acknowledgments: We would like to express our gratitude to all members of the Elmer FEM team for their efforts and intensive work on Elmer FEM software development.

Conflicts of Interest: The authors declare no conflict of interest.

\section{References}

1. Meydan, T.; Overshott, K.J. Amorphous force transducers in ac applications. J. Appl. Phys. 1982, 51, 8383-8385. [CrossRef]

2. Bieńkowski, A.; Kulikowski, J. The magnetoelastic Villari effect in ferrites. J. Magn. Magn. Mater. 1980, 19, 120-122. [CrossRef]

3. Bieńkowski, A.; Szewczyk, R. The possibility of utilizing the high permeability magnetic materials in construction of magnetoelastic stress and force sensors. Sens. Actuators A Phys. 2004, 113, 270-276. [CrossRef]

4. Williams, S.R. Some Experimental Methods in Magnetostriction. JOSA 1927, 14, 383-408. [CrossRef]

5. Villari, E. Change of magnetization by tension and by electric current. Annu. Rev. Phys. Chem. 1865, 126, 87-122. [CrossRef]

6. Liberal, I.; Engheta, N. Near-zero refractive index photonics. Nat. Photonics 2017, 11, 149-158. [CrossRef]

7. Spada, L.; Spooner, C.; Haq, S.; Hao, Y. Curvilinear MetaSurfaces for Surface Wave Manipulation. Sci. Rep. 2019, 9, 3107. [CrossRef]

8. Dahle, O. The pressductor and the torductor-Two heavy-duty transducers based on magnetic stress sensitivity. IEEE Trans. Commun. Electron. 1964, 83, 752-758. [CrossRef]

9. Oess, N.P.; Weisse, B.; Nelson, B.J. Magnetoelastic Strain Sensor for Optimized Assessment of Bone Fracture Fixation. IEEE Sens. J. 2009, 9, 961-968. [CrossRef]

10. Herzer, G. Magnetoelastic Sensors for Electronic Article Surveillance. Sens. Lett. 2007, 5, 259-262. [CrossRef]

11. Salach, J.; Szewczyk, R.; Bieńkowski, A.; Jackiewicz, D. Analysis and computer modeling of magnetoelastic characteristics of FeNi-based amorphous ring-shaped core under uniform compressive and tensile stresses. Theor. Appl. Inform. 2013, 25, 29-40. [CrossRef]

12. Szewczyk, R.; Bieńkowski, A. Stress dependence of sensitivity of fluxgate sensor. Sens. Actuators A Phys. 2004, 110, 232-235. [CrossRef]

13. Ktena, A.; Hristoforou, E. Stress Dependent Magnetization and Vector Preisach Modeling in Low Carbon Steels. IEEE Trans. Magn. 2012, 48, 1433-1436. [CrossRef]

14. Lahyaoui, O.; Lanfranchi, V.; Buiron, N.; Martin, F.; Aydin, U.; Belahcen, A. Effect of mechanical stress on magnetization and magnetostriction strain behavior of non-oriented Si-Fe steels at different directions and under pseudo-DC conditions. Int. J. Appl. Electromagn. Mech. 2019, 60, 1-14. [CrossRef]

15. Sablik, M.J.; Jiles, D.C. Coupled magnetoelastic theory of magnetic and magnetostrictive hysteresis. IEEE Trans. Magn. 1993, 29, 2113-2123. [CrossRef]

16. Yang, B.; Li, H.; Zhang, A. Influences of non-coaxial magnetic field on magneto-mechanical effect of ferromagnetic steel. Int. J. Appl. Electromagn. Mech. 2018, 59, 1-8. [CrossRef]

17. Nowicki, M. Tensductor-Amorphous Alloy Based Magnetoelastic Tensile Force Sensor. Sensors 2018, 18, 4420. [CrossRef]

18. Stefanescu, D.M. Handbook of Force Transducers: Principles and Components; Springer Science \& Business Media: Berlin, Germany, 2011; ISBN 3642182968.

19. Szewczyk, R.; Bieńkowski, A. Magnetoelastic Villari effect in high-permeability Mn-Zn ferrites and modeling of this effect. J. Magn. Magn. Mater. 2003, 254, 284-286. [CrossRef]

20. Bieńkowski, A.; Szewczyk, R.; Salach, J. Industrial application of magnetoelastic force and torque sensors. Acta Phys. Pol. A 2010, 118, 1008-1009. [CrossRef]

21. Sablik, M.; Rubin, S.; Riley, L.; Jiles, D.; Kaminski, D.; Biner, S. A model for hysteretic magnetic properties under the application of noncoaxial stress and field. J. Appl. Phys. 1993, 74, 480-488. [CrossRef]

22. Kwun, H. Investigation on the dependence of barkhausen noise on stress and the angle between the stress and magnetization direction. J. Magn. Magn. Mater 1985, 49, 235-240. [CrossRef]

23. Rekik, M.; Hubert, O; Daniel, L. Influence of a multiaxial stress on the reversible and irreversible magnetic behaviour of a 3\%Si-Fe alloy. Int. J. Appl. Electromagn. Mech. 2014, 44, 301-315. [CrossRef] 
24. Timoshenko, S.P.; Goodier, J.N. Theory of Elasticity, 3rd ed.; McGraw-Hill International Editions: New York, NY, USA, 1970.

25. Goldstein, H.; Poole, C.P.; Safko, J. Classical Mechanics, 3rd ed.; Addison Wesley: Boston, MA, USA, 2002; pp. 782-783.

26. Seddik, H.; Greve, R.; Zwinger, T.; Gillet-Chaulet, F.; Gagliardini, O. Simulations of the Greenland ice sheet 100 years into the future with the full Stokes model Elmer/Ice. J. Glaciol. 2012, 58, 427-440. [CrossRef]

27. Schöberl, J. NETGEN-An advancing front 2D/3D-mesh generator based on abstract rules. Comput. Vis. Sci. 1997, 1, 41-52. [CrossRef]

28. Vacuumschmelze Information Sheets MSDS VITROPERM. Available online: https://www.vacuumschmelze. com/en/the-company/quality/information-sheets-msds/vitroperm.html (accessed on 4 November 2018).

29. Chang, C.; Inoue, A. FeNi-based bulk glassy alloys with superhigh mechanical strength and excellent soft-magnetic properties. Appl. Phys. Lett. 2006, 89, 051912. [CrossRef]

30. Bieńkowski, A. Some problems of measurement of magnetostriction in ferrites under stresses, J. Magn. Magn. Mater. J. Magn. Magn. Mater. 1992, 112, 143-145. [CrossRef]

(C) 2019 by the authors. Licensee MDPI, Basel, Switzerland. This article is an open access article distributed under the terms and conditions of the Creative Commons Attribution (CC BY) license (http://creativecommons.org/licenses/by/4.0/). 